Check for updates

Cite this: Mater. Chem. Front., 2020, 4, 1397

Received 12th December 2019 Accepted 17th January 2020

DOI: 10.1039/c9qm00753a

rsc.li/frontiers-materials

\title{
Intervaginal space injection of a liquid metal can prevent breast cancer invasion and better-sustain concomitant resistance
}

\author{
Yupeng Cao, ${ }^{\text {ab }}$ Xiajun Hu, ${ }^{c}$ Qiang Zhang, ${ }^{a}$ Wenda Hua, ${ }^{a}$ Nan Hu, ${ }^{\text {abd }}$ Yifeng Nie, ${ }^{\text {ab }}$ \\ Xue $\mathrm{Xu},{ }^{e}$ Yonggang $\mathrm{Xu}^{\mathrm{f}}{ }^{\mathrm{c}}$ Chongqing Yang, ${ }^{\mathrm{g}}$ Xiaohan Zhou, ${ }^{\mathrm{ab}}$ Wentao Liu*a and \\ Dong Han (D) *ab
}

\begin{abstract}
Chest wall (CW) invasion by recurrent or locally advanced breast cancer (BC) requires further treatment with more potential complications. The negative margin of a tumor has been proposed as a critical factor in BC surgery; however, the ideal margin remains controversial, and data suggest that this parameter cannot further reduce CW invasion. Herein, a liquid metal (LM) was introduced as an interstitial stream (IS) to separate the BC and CW to prevent tumor invasion into the CW. Successful continuous separation and the prevention of CW invasion were observed in 4T1 tumor-bearing mice by magnetic resonance imaging (MRI) and anatomical observation, respectively. Furthermore, the inhibition of primary tumor growth and the prevention of accelerated secondary tumor growth suggest that this method potently retains concomitant resistance (CR). Subsequently, the enhanced maturation of dendritic cells (DCs) from the LM group suggests that this method may activate $T$ cell-dependent immunity to retain CR through DC maturation. Additionally, LM was shown to have limited toxicity. In this study, LM was introduced as an IS into the interstitial space, which potentially links superficial and deep sites in the body, and showed excellent ability to prevent CW invasion, thus also providing a potential way to efficiently deliver drugs.
\end{abstract}

\section{Introduction}

Breast cancer (BC) is one of the most common cancers in females worldwide. ${ }^{1,2}$ Depending on the stage of BC at diagnosis, numerous therapeutic options are currently available, including surgery, chemotherapy, radiation therapy and immunotherapy. ${ }^{3}$ For early-stage BC, breast conserving therapy (BCT) is commonly performed as a surgical treatment, ${ }^{4,5}$ but this treatment strategy can fail, leading to local recurrence. ${ }^{6}$ Recurrences in the chest wall

\footnotetext{
${ }^{a}$ CAS Center for Excellence in Nanoscience, National Center for Nanoscience and Technology, Beijing 100190, China. E-mail: dhan@nanoctr.cn, liuwentao@nanoctr.cn

${ }^{b}$ School of Future Technology, University of Chinese Academy of Sciences, Beijing 100049, China

${ }^{c}$ Department of Cardiology, Union Hospital, Tongji Medical College, Huazhong University of Science and Technology, Wuhan 430022, China

${ }^{d}$ Department of Traditional Chinese Medicine, Chengde Medical University, Chengde 066000, China

${ }^{e}$ Department of Gerontology, The Second Hosipital of Shandong University, Jinan, China

${ }^{f}$ Department of Hematology, Xiyuan Hospital, China Academy of Chinese Medical Sciences, Beijing 100091, China

${ }^{g}$ Department of Pathology, Beijing Hospital, Beijing 100730, China
}

(CW) require further treatment, ${ }^{2}$ as does locally advanced BC involving the CW. ${ }^{3}$ However, these further treatments are associated with more complications. For instance, pulmonary and infectious complications following $\mathrm{CW}$ resection and reconstruction have been reported. ${ }^{7}$ Therefore, avoiding CW invasion is critical for obtaining a favorable prognosis.

The negative margin width for the surgical resection of $\mathrm{BC}$ is related to the risk of recurrence. ${ }^{8}$ To reduce recurrence involving the $\mathrm{CW}$, many studies have focused on methods for the intraoperative determination of tumor margins, including magnetic resonance imaging (MRI), computed tomography and invisible near-infrared fluorescent light imaging. ${ }^{9-13}$ In addition, treatment of locally advanced BC that has not invaded the $\mathrm{CW}$ requires recognizing the negative tumor margins. ${ }^{3}$ However, the optical negative margin width is still controversial regarding minimizing the recurrence risk after the surgical resection of BC. ${ }^{8,14-16}$ More effective methods are needed to reduce CW invasion. In recent years, the biological utilisation of the intervaginal space in medical applications has been emerging. ${ }^{17-20}$ In particular, a previous study on the flow behavior of LMs in the connected fascial space (FS) showed that the IS in the FS system is a potential pathway to deliver drugs targeting certain regions of the body. ${ }^{19}$ 
In this study, we introduced an LM, a gallium-based alloy, as an obstruction to reduce the $\mathrm{CW}$ invasion of $\mathrm{BC}$ in breast tumor-bearing mice. Intervaginal space injection (ISI) of a LM into the wrist was performed in tumor-bearing mice. MRI was used to observe the obstructive effect of the LM. Gross dissection was performed to detect the ability of this method to block CW invasion. In addition, the potential immunological effect of this method was observed by flow cytometry (FCM). Histological sections of various viscera were examined to confirm the safety of LM in this study.

\section{Experimental}

\section{ISI in tumor-bearing mice}

A gallium-based alloy composed of $75 \%$ gallium and $25 \%$ indium melted at $15{ }^{\circ} \mathrm{C}$ was provided by the Technical Institute of Physics and Chemistry at the Chinese Academy of Sciences. Female Balb/c mice (with a body weight of approximately $20 \mathrm{~g}$ ) were purchased from Beijing Vital River Experimental Animal Corporation (Beijing, China). The 4T1 BC cell line used in this study was purchased from the Type Culture Collection (Chinese Academy of Sciences, Shanghai, China).

All the animal experiments were approved by the Committee at the Institute of the Chinese Academy of Medical Science. 4T1 cells were cultured in Dulbecco's modified Eagle's medium supplemented with $10 \%$ fetal bovine serum (Gibco), $100 \mathrm{U} \mathrm{mL}^{-1}$ penicillin, and $1 \mathrm{mg} \mathrm{mL}^{-1}$ streptomycin at $37{ }^{\circ} \mathrm{C}$ in an incubator with $95 \%$ air and $5 \% \mathrm{CO}_{2}$. $4 \mathrm{~T} 1$ cells $\left(1 \times 10^{6}\right)$ suspended in PBS were subcutaneously injected into the left flank of each female BALB/c mouse. On the seventh day, 4 T1 cells $\left(2 \times 10^{5}\right)$ suspended in PBS were injected into the right flank of each female BALB/c mouse. The tumor volume was calculated according to the following formula: width ${ }^{2} \times$ length $\times 0.5$. The tumor volume at a specific time point divided by the tumor volume on day 8 is reported. On the eighth day, each mouse in the LM group underwent ISI with LM $(0.1 \mathrm{~mL})$, and each mouse in the PBS group underwent ISI with PBS $(0.1 \mathrm{~mL})$. The control group was not subjected to ISI.

\section{In vivo MRI}

The mice were observed by MRI (Bruker, 7T) before and after ISI on day 8. In addition, MRI was performed on day 12 . $T_{2}$-weighted MRI was used with the following parameters: $\mathrm{TR}=2000 \mathrm{~ms} ; \mathrm{TE}=30 \mathrm{~ms} ; \mathrm{FOV}=28 \times 28 \mathrm{~mm}^{2} ;$ and image size $=256 \times 256$.

\section{Ex vivo analysis of DCs in the spleen}

To study DC maturation, the spleens were harvested from the mice and stained with anti-CD11c-PE (Biolegend, Clone: N418, Catalog: 117307), anti-CD86-APC (Biolegend, Clone: GL-1, Catalog: 105011), and anti-CD80-Alexa Fluor ${ }^{\circledR} 488$ (Biolegend, Clone: 16-10A1, Catalog: 104715) antibodies according to the manufacturer's protocols. A Beckman Coulter Navios flow cytometer system (USA) was used. The data were analyzed by the flow cytometer workstation.

\section{Histopathological analysis}

This analysis aimed to examine the potential toxicity of LM in mice. Heart, liver, spleen, lung and kidney tissues were harvested from the mice and fixed overnight in $4 \%$ neutralbuffered paraformaldehyde. The histological slides were stained with hematoxylin and eosin ( $\mathrm{HE}$ ), followed by dehydration through a graded series of ethanol solutions from $75 \%$ to $100 \%$, and examined in a blinded manner by a well-trained pathologist. Histological observations and photomicrography were performed using a digital slide scanner (NanoZoomer-XR C12000, Japan). Damage to the above visceral tissues was examined in an average of five fields of view $(\times 200)$.

\section{Results and discussion}

To directly assess the separation effect of LM, we utilized $T_{2}$-weighted MRI to observe the positions of the tumor and $\mathrm{CW}$ in mice. Because the transverse relaxation $\left(T_{2}\right)$ value of tumors is high in this paper, the signal of the tumors was high in the $T_{2}$-weighted MRI. Because the $T_{2}$ value of PBS is higher than that of tumors, the signal of PBS is higher than that of the tumor in the $T_{2}$-weighted MRI. ${ }^{21}$ Before ISI, the tumor exhibited high signal intensity. Hydrogen in mice was used for visualization by MRI as LM does not contain hydrogen. Hence, we can observe LM in mice through its low signal intensity, as shown in Fig. 1b. In addition, a portion of the injected LM rapidly entered the region between the tumor and CW within ten minutes after ISI. Similarly, phosphate-buffered saline (PBS) was distributed in the region between the tumor and CW. The results suggest that fluids, including PBS and LM, can flow through the FS to the region near a tumor.

LM outperformed PBS in mice in this study. Specifically, the mobilities of these two agents were different. LM distributed more in the region between the tumor and the CW, while PBS distributed in the region between the tumor and arm. Furthermore, LM remained in the mice longer than PBS. As shown in Fig. 1b, LM remained at four days after injection, but PBS could not be observed at this time point. PBS is a waterbased solution and hence can be absorbed by mice. It is difficult for LM to diffuse throughout the body due to its high surface tension. ${ }^{19}$ Therefore, LM has the potential to remain in a region in mice for a long period and resist tumor invasion. Subsequently, we compared the separation effect between the LM and blank control groups. As shown in Fig. 2, LM remained between the CW and the tumor. However, the tumors in the other two groups grew adjacent to CW tissues. As evidenced by tumors explanted during the growth period, LM and PBS did not flow to the region near the tumor on the opposite flank. This result suggests that LM is limited to a certain region in tumor-bearing mice.

Dissection was performed to examine the ability of LM to separate the tumor and the CW. At the molecular level, the invasion of the tumor involves the tumor cells secreting enzymes to degrade the extracellular matrix adjacent to the tumor. At the tissue level, invasion refers to the direct extension 
a
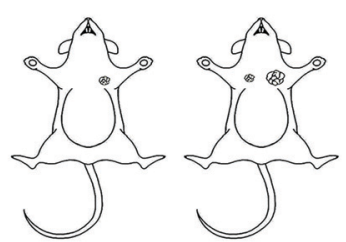

\begin{tabular}{cc} 
Day 1 & Day 7 \\
\hline $\begin{array}{l}\text { First tumor } \\
\text { inoculation }\end{array}$ & $\begin{array}{c}\text { Second tumor } \\
\text { inoculation }\end{array}$
\end{tabular}

b
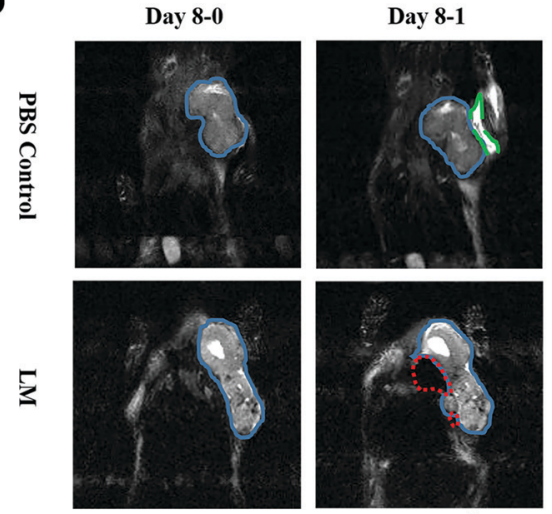

() PBS

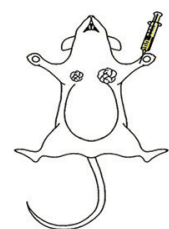

Day 8

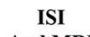

And MRI

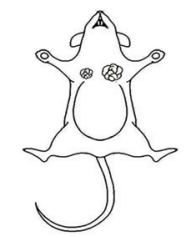

MRI

Observation

Fig. 1 Study course and MRI of the LM and PBS groups. The green dashed line represents PBS, the blue full lines indicate the tumor, the red dotted lines represent LM, and days 8-0 and 8-1 indicate images taken before and after ISI, respectively. (a) The study course, including the first and second tumor inoculations, ISI with LM and MRI. (b) Observation of mice in the LM and PBS groups before and after ISI with LM and on the following 4th day. The low signal intensity representing LM was distributed in the region between the tumor and the CW, whereas PBS was distributed near the tumor after ISI, but disappeared on the following 4th day.

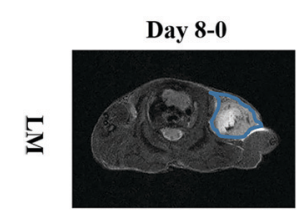

Day 8

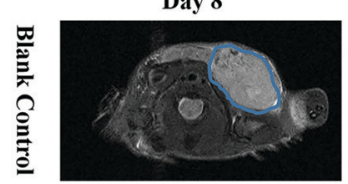

Fig. $2 \mathrm{MRI}$ observations of the LM and blank control groups. LM remaining in the targeted region is indicated by red dotted lines and separates the CW and the tumor, which is indicated by blue full lines.

and penetration by cancer cells into neighboring tissues. The barrier between the tumor and the chest wall muscle was breached. ${ }^{22}$ As shown in Fig. 3, CW invasion occurred in mice in the control group. The muscles of the $\mathrm{CW}$ adjacent to the breast tumor were seriously damaged in the control group, and the tumor margin was ambiguous. Compared to the control group, the LM group exhibited better separation of the tumor margin.

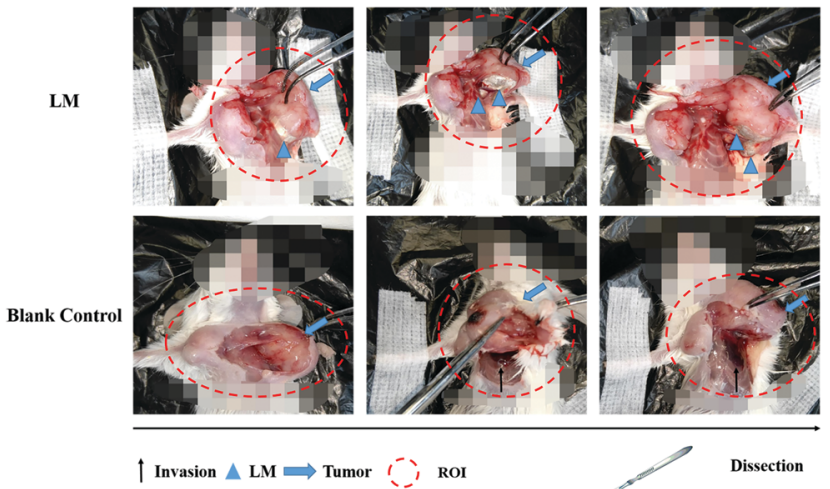

Fig. 3 Anatomical observation of mice in the LM and control groups upon surgical dissection. The black arrows represent invasion regions, the blue triangles represent LM, the blue arrows represent tumors and the red dashed lines represent the region of interest (ROI); mosaics were used to cover the heads and abdomens of the mice. LM separated the tumor and the $\mathrm{CW}$; however, CW invasion occurred in mice of the control group.

After dissection, LM was found to have remained in the region between the tumor and the $\mathrm{CW}$, and the CW muscles were not damaged. Importantly, LM was enwrapped by a film of fascia, a type of interstitial tissue. After ISI, LM flowed from the injection site and continuously distributed in the region between the tumor and the CW through the FS. Direct tumor invasion disrupting the fascia film has been reported. ${ }^{23}$ In addition, tumor invasion occurring on the adjacent normal tissues due to the acidic microenvironment has been reported. ${ }^{24}$ Because of the acidic environment, the galliumbased liquid metal might be degraded to form gallium(III) ions, which can be used as an anticancer agent. ${ }^{25}$ In this study, LM was shown to play a role in separating the tumor and the $\mathrm{CW}$ and hence resisting the $\mathrm{CW}$ invasion by the tumor. Due to the direct physical separation created by LM, the tumor cells cannot invade fascia films and other tissues. Moreover, as LM localized adjacent to the tumor, the tumor margin was distinct, which facilitates tumor recognition. In addition, the fascia enwrapping the $\mathrm{CW}$ remained smooth because it was not invaded. Hence, the fascia enwrapping the breast tumor was easily dissected, which will benefit BCT.
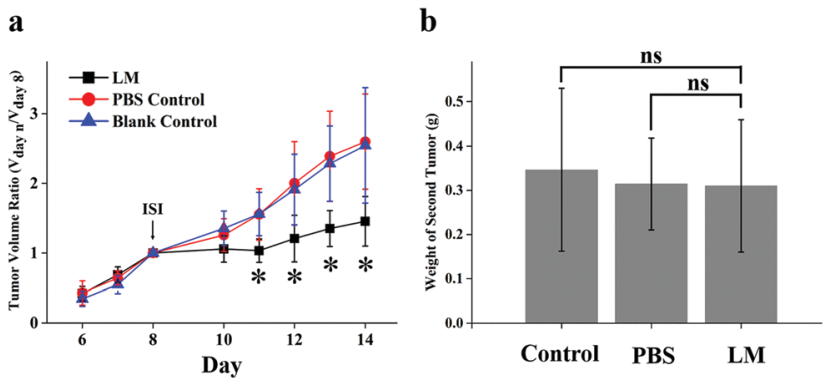

Fig. 4 Tumor growth analysis. (a) The primary tumor volumes in the three groups were plotted. The primary tumor volumes on days 11 to 14 in the LM group were significantly lower than those in the other groups. (b) The secondary tumor weights in the three groups were not significantly different. $p$ values were determined with the $t$-test $\left({ }^{\star} p<0.05\right)$. 


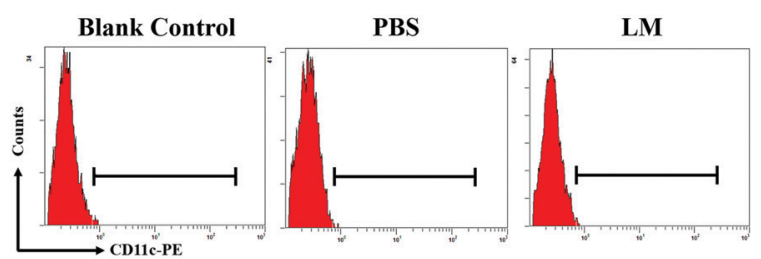

b
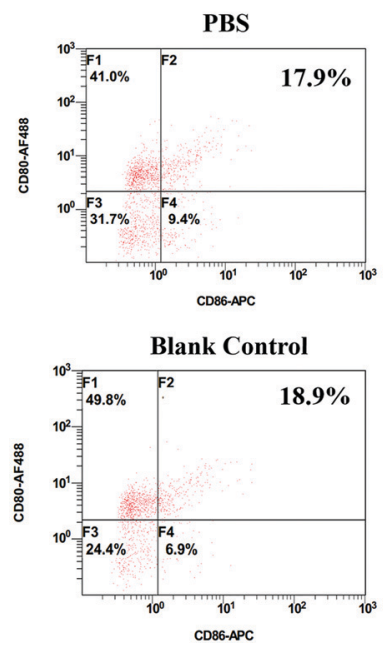
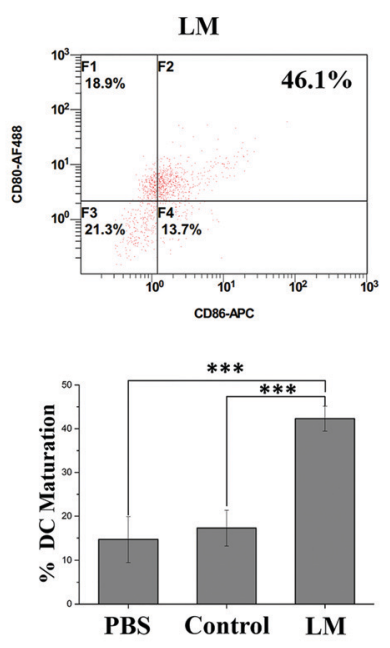

Fig. 5 DC maturation. (a) Identification of DCs by CD11c using FCM (b) DC maturation in the three groups was plotted according to CD11C, CD80 and CD86 expressions. DC maturation in the LM group was significantly increased compared to the other groups. $p$ values were determined with the $t$-test $\left({ }^{* \star} p<0.001\right)$.

Relapse after surgery results in worse patient outcomes. However, in experimental and clinical settings, primary tumor dissection has been shown to contribute to the accelerated growth of spontaneous metastases. ${ }^{26-28}$ This phenomenon, the inhibition of metastases by the primary tumor, is called concomitant resistance (CR) and is relieved by dissection of the primary tumor. ${ }^{26,29}$ Hence, the reduction in CR of metastatic foci after treatment should be taken into consideration. In this experiment, secondary tumor weight and primary tumor growth were compared in the three groups. As shown in Fig. 4a,

the volume of the primary tumor was significantly lower in the LM group than in the PBS and control groups from day 11 to day 14 after ISI. In addition, the secondary tumors were not heavier in the LM group than in the other two groups, as shown in Fig. 4b. Therefore, LM inhibited primary tumor growth and concurrently retained potent CR.

$\mathrm{T}$ cell-dependent immunity plays a pivotal role in $\mathrm{CR} .^{30}$ As one of the most important classes of antigen-presenting cells, DCs play crucial roles in initiating and regulating $\mathrm{T}$ celldependent immunity. ${ }^{31}$ CD80 and CD86 molecules expressed on dendritic cells are co-stimulatory molecules known for their role in the activation of T-cell. CD80 is downregulated on most of the cancer cells. In addition, the loss of CD80 is sufficient to allow cancer cells to escape the attack of the immune system and to impart anergy and apoptosis in tumor-infiltrating $\mathrm{T}$ cells. CD86 is responsible for delivering additional or second signals to $\mathrm{T}$ cells when it interacts with the ligand CD28. Upregulating the expression of co-stimulatory molecules such as CD80 and CD86 and the secreted pro-inflammatory cytokines of DCs contribute to the presentation of antigens to T cells. ${ }^{32}$ And this enhanced immune effect might further contribute to the sustainment of CR. The immune effects of DC induced by mechanical stimuli have been reported; for instance, DC activation by increased extracellular pressure and changes in DC activity based on substrate stiffness. ${ }^{33-35}$ In this study, the heavy LM not only separated the tumor and the CW but also potentially stretched the fascia enwrapping the tumor due to its presence and weight. Hence, this stretch force may have activated immune responses in the mice. The expression of co-stimulatory molecules (CD80 and CD86), which are the hallmarks of DC maturation, is a critical factor that determines the quality of adaptive immunity. ${ }^{36}$ Therefore, we examined DC maturation in the spleens of mice in the three groups by analyzing CD80 and CD86 expression by FCM. As shown in Fig. 5, CD80 and CD86 expressions on DCs were higher in the LM group than in the other two groups. Hence, the DC maturation rate was significantly increased in the LM group compared to the other two groups.

These results suggest that LM introduced as an IS may activate DC maturation through effects on fascia space and further
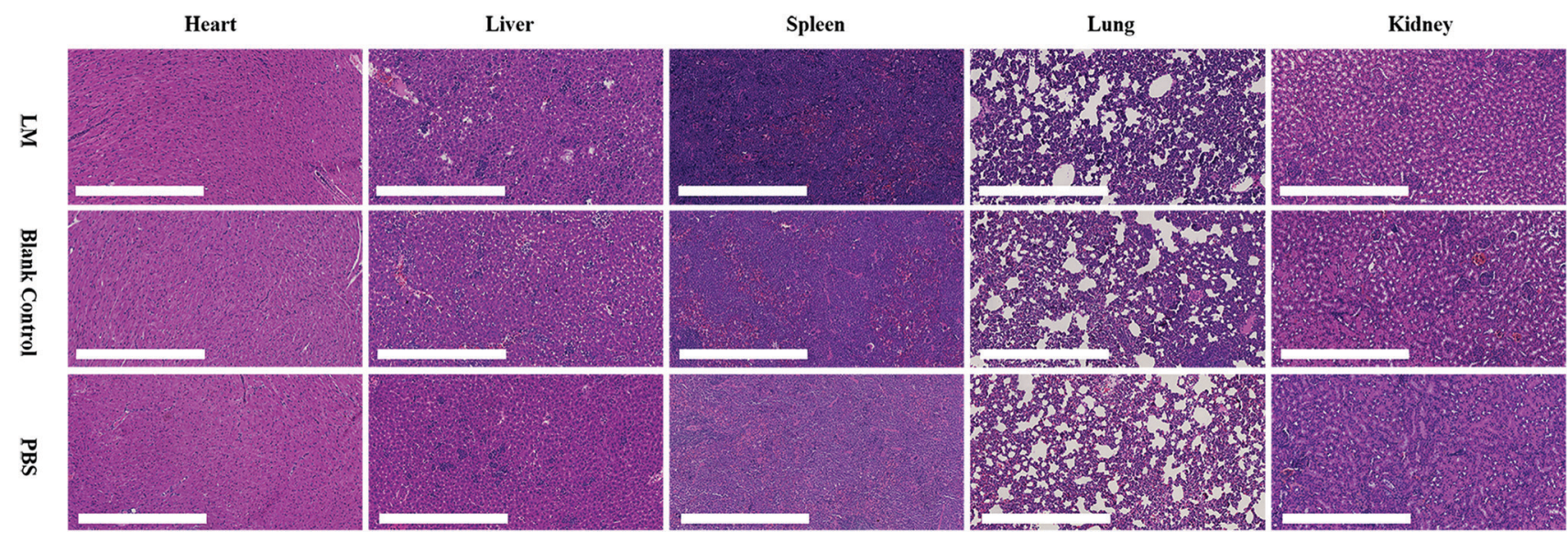

Fig. 6 Histopathological examination of the hearts, livers, spleens, lungs and kidneys from mice in the LM, blank control and PBS control groups. 
prevent the accelerated growth of metastases after inhibiting primary tumor growth. The combination of enhanced DC maturation and checkpoint blockade therapy significantly inhibited tumor growth. ${ }^{37}$ Hence, this method has the potential to prevent the growth of metastases caused by a reduction in CR and enhance the immune response in combination with checkpoint blockade therapy.

LM is inorganic matter with reportedly low toxicity. ${ }^{38}$ However, the dose of LM used in this study was quite high for mice. We performed a histological examination to evaluate the toxicity of LM. As shown in Fig. 6, vacuolar degeneration, cytoplasm swelling and degeneration and inflammation occurred in the heart, liver and lung tissues of mice in all three groups. The spleen and kidney histological results revealed no abnormalities. Although some negative effects occurred, the histological results did not suggest that LM impaired the viscera of mice.

To avoid the risk of toxicity from LM, alternative materials that satisfy the aforementioned features and show appropriate biosafety and biocompatibility may be a better choice to facilitate the clinical application of this method. As suggested by a previous study on ISs ${ }^{19}$ and this work, the materials injected in the FS must have three features: mobility, the ability to diffuse into tissues, and the ability to resist degradation. For instance, collagen and hyaluronic acid used in the clinic have the potential to replace the liquid metal. Additionally, loading anticancer drug onto the substitute may be a more efficient and effective treatment. ${ }^{39}$

\section{Conclusions}

In summary, we have described a method of introducing LM as an IS injection into the FS to efficiently link superficial to deep sites and to successfully separate the CW and the BC in tumor-bearing mice. LM flowed through the FS under the injection site to the region between the primary tumor and the CW. During the experimental period, LM did not flow to the region between the secondary tumor and the CW. LM remained for a long period and continuously resisted CW invasion by the primary tumor. Based on the primary tumor growth and secondary tumor weight results, the impact of this method on CR was discussed. Subsequently, the significant increase in DC maturation in the spleens from mice in the LM group suggests that sustained CR may stem from T cell-dependent immunity regulated by DCs. In addition, the combination of this method and checkpoint blockade as a potential therapy was proposed for the treatment of BC, not only as a preventative strategy for CW invasion. Additionally, the advantages and disadvantages of LM were discussed. Additional potential replacement materials were also discussed. This method utilized the interstitial space, which is between two adjacent interstitial tissues, as a pathway for targeted delivery to a particular region. The agents can be injected at a superficial site and flow through the interstitial space to deep within the body, exhibiting highly efficient passage from superficial sites to specific deep regions. More investigations on the IS, i.e., the link between superficial sites and deep sites, will be of great value and facilitate the development of therapeutics that will contribute to human health.

\section{Author contributions}

Dong Han and Wentao Liu designed research, analyzed data, and wrote the paper. Yupeng Cao analyzed data and wrote the paper. Xiajun $\mathrm{Hu}$, Qiang Zhang, Wenda Hua, Nan Hu, Yifeng $\mathrm{Nie}$ and Xue $\mathrm{Xu}$ performed the anatomy experiment. Yupeng Cao performed the magnetic resonance imaging experiment. Yonggang $\mathrm{Xu}$ and Yupeng Cao performed the flow cytometer experiment. Congqing Yang analyzed the histopathological data. Xiaohan Zhou performed the mouse tumor model.

\section{Conflicts of interest}

There are no conflicts to declare.

\section{Acknowledgements}

We are sincerely thankful to Technical Institute of Physics and Chemistry at the Chinese Academy of Sciences for providing the liquid metal. This work was supported by the Source Innovation Research Program of Frontier Sciences, CAS, Grant No. ZDBSLY-SLH036.

\section{Notes and references}

1 B. W. Stewart and C. P. Wild, World Cancer Report 2014, International Agency for Research on Cancer, 2014.

2 E. Wakeam, S. A. Acuna and S. Keshavjee, Chest wall resection for recurrent breast cancer in the modern era a systematic review and meta-analysis, Ann. Surg., 2018, 267, 646.

3 B. Sepesi, Management of breast cancer invading chest wall, Thorac. Surg. Clin., 2017, 27, 158.

4 M. Morrow, R. A. Schmidt and C. Bucci, Breast conservation for mammographically occult carcinoma, Ann. Surg., 1998, 227, 502.

5 M. R. Grootendorst, M. Cariati, S. E. Pinder, A. Kothari, M. Douek, T. Kovacs, H. Hamed, A. Pawa, F. Nimmo, J. Owen, V. Ramalingam, S. Sethi, S. Mistry, K. Vyas, D. S. Tuch, A. Britten, M. Van Hemelrijck, G. J. Cook, C. Sibley-Allen, S. Allen and A. Purushotham, Intraoperative assessment of tumor resection margins in breast-conserving surgery using F-18-FDG cerenkov luminescence imaging: A first-inhuman feasibility study, J. Nucl. Med., 2017, 58, 891.

6 M. Clemons, S. Danson, T. Hamilton and P. Goss, Locoregionally recurrent breast cancer: incidence, risk factors and survival, Cancer Treat. Rev., 2001, 27, 67.

7 J. D. Spicer, J. B. Shewale, M. B. Antonoff, A. M. Correa, W. B. Hofstetter, D. C. Rice, A. A. Vaporciyan, R. J. Mehran, G. L. Walsh, J. A. Roth and B. Sepesi, The influence of reconstructive technique on perioperative pulmonary and 
infectious outcomes following chest wall resection, Ann. Thorac. Surg., 2016, 102, 1653.

8 M. Pilewskie and M. Morrow, Margins in breast cancer: How much is enough?, Cancer, 2018, 124, 1335.

9 P. L. Kubben, K. J. ter Meulen, O. E. M. G. Schijns, M. P. ter Laak-Poort, J. J. van Overbeeke and H. van Santbrink, Intraoperative MRI-guided resection of glioblastoma multiforme: a systematic review, Lancet Oncol., 2011, 12, 1062.

10 S. Gioux, H. S. Choi and J. V. Frangioni, Image-guided surgery using invisible near-infrared light: fundamentals of clinical translation, Mol. Imaging, 2010, 9, 237.

11 A. L. Vahrmeijer, M. Hutteman, J. R. van der Vorst, C. J. H. van de Velde and J. V. Frangioni, Image-guided cancer surgery using near-infrared fluorescence, Nat. Rev. Clin. Oncol., 2013, 10, 507.

12 Z. Lei, X. Li, X. Luo, H. He, J. Zheng, X. Qian and Y. Yang, Bright, stable, and biocompatible organic fluorophores absorbing/emitting in the deep near-infrared spectral region, Angew. Chem., Int. Ed., 2017, 56, 2979.

13 Q. X. Wen, Y. J. Zhang, C. Y. Li, S. S. Ling, X. H. Yang, G. C. Chen, Y. Yang and Q. B. Wang, NIR-II fluorescent selfassembled peptide nanochain for ultrasensitive detection of peritoneal metastasis, Angew. Chem., Int. Ed., 2019, 58, 11001.

14 U. Veronesi, N. Cascinelli, L. Mariani, M. Greco, R. Saccozzi, A. Luini, M. Aguilar and E. Marubini, Twenty-year follow-up of a randomized study comparing breast-conserving surgery with radical mastectomy for early breast cancer, N. Engl. J. Med., 2002, 347, 1227.

15 J. A. Vandongen, H. Bartelink, I. S. Fentiman, T. Lerut, F. Mignolet, G. Olthuis, E. Vanderschueren, R. Sylvester, D. Tong, J. Winter and K. Vanzijl, Factors influencing local relapse and results of salvage treatment after breastconserving therapy in operable breast-cancer - Eortc Trial10801, breast conservation compared with mastectomy in TNM stage-I and stage-II breast-cancer, Eur. J. Cancer, 1992, 28A, 801.

16 B. Fisher, J. Dignam, N. Wolmark, E. Mamounas, J. Costantino, W. Poller, E. R. Fisher, D. L. Wickerham, M. Deutsch, R. Margolese, N. Dimitrov and M. Kavanah, Lumpectomy and radiation therapy for the treatment of intraductal breast cancer: Findings from national surgical adjuvant breast and bowel project B-17, J. Clin. Oncol., 1998, 16, 441 .

17 J. Feng, F. Wang, X. Han, Z. Ao, Q. Sun, W. Hua, P. Chen, T. Jing, H. Li and D. Han, A "green pathway" different from simple diffusion in soft matter: fast molecular transport within micro/nanoscale multiphase porous systems, Nano Res., 2014, 7, 434.

18 X. Shi, Y. Zhu, W. Hua, Y. Ji, Q. Ha, X. Han, Y. Liu, J. Gao, Q. Zhang, S. Liu, K. Ren, X. Wu, H. Li and D. Han, An in vivo study of the biodistribution of gold nanoparticles after intervaginal space injection in the tarsal tunnel, Nano Res., 2016, 9, 2097.

19 N. Hu, Y. Cao, Z. Ao, X. Han, Q. Zhang, W. Liu, S. Liu, F. Liao and D. Han, Flow behavior of liquid metal in the connected fascial space: Intervaginal space injection in the rat wrist and mice with tumor, Nano Res., 2018, 11, 2265.

20 Y. Cao, Y. Zhang, Y. Wang, W. Liu and D. Han, Improved stimulated echo in diffusion magnetic resonance imaging: introducing a pi pulse for SNR enhancement, Magn. Reson. Med., 2019, 81, 2905.

21 M. C. Maas, E. K. Vos, M. W. Lagemaat, A. K. Bitz, S. Orzada, T. Kobus, O. Kraff, S. Maderwald, M. E. Ladd and T. W. J. Scheenen, Feasibility of T-2-weighted turbo spin echo imaging of the human prostate at 7 tesla, Magn. Reson. Med., 2014, 71, 1711.

22 E. A. McSherry, S. Donatello, A. M. Hopkins and S. McDonnell, Molecular basis of invasion in breast cancer, Cell. Mol. Life Sci., 2007, 64, 3201.

23 M. Polette, B. Nawrocki-Raby, C. Gilles, C. Clavel and P. Birembaut, Tumour invasion and matrix metalloproteinases, Crit. Rev. Oncol. Hematol., 2004, 49, 179.

24 V. Estrella, T. Chen, M. Lloyd, J. Wojtkowiak, H. H. Cornnell, A. Ibrahim-Hashim, K. Bailey, Y. Balagurunathan, J. M. Rothberg, B. F. Sloane, J. Johnson, R. A. Gatenby and R. J. Gillies, Acidity generated by the tumor microenvironment drives local invasion, Cancer Res., 2013, 73, 1524.

25 P. Collery, B. Keppler, C. Madoulet and B. Desoize, Gallium in cancer treatment, Crit. Rev. Oncol. Hematol., 2002, 42, 283.

26 R. A. Ruggiero, J. Bruzzo, P. Chiarella, O. D. Bustuoabad, R. P. Meiss and C. D. Pasqualini, Concomitant tumor resistance: The role of tyrosine isomers in the mechanisms of metastases control, Cancer Res., 2012, 72, 1043.

27 C. M. Galmarini, O. Tredan and F. C. Galmarini, Concomitant resistance and early-breast cancer: should we change treatment strategies?, Cancer Metastasis Rev., 2014, 33, 271.

28 R. Demicheli, M. W. Retsky, W. J. M. Hrushesky and M. Baum, Tumor dormancy and surgery-driven interruption of dormancy in breast cancer: learning from failures, Nat. Clin. Pract. Oncol., 2007, 4, 699.

29 R. A. Ruggiero, O. D. Bustuoabad, R. D. Bonfil, R. P. Meiss and C. D. Pasqualini, Concomitant immunity in murine tumors of non-detectable immunogenicity, Br. J. Cancer, 1985, 51, 37.

30 B. Quesnel, Tumor dormancy and immunoescape, APMIS, 2008, 116, 685-694.

31 C. A. Janeway and K. Bottomly, Signals and signs for lymphocyte-responses, Cell, 1994, 76, 275.

32 D. M. Sansom, C. N. Manzotti and Y. Zheng, What's the difference between CD80 and CD86?, Trends Immunol., 2003, 24, 314 .

33 S. F. B. Mennens, M. Bolomini-Vittori, J. Weiden, B. Joosten, A. Cambi and K. van den Dries, Substrate stiffness influences phenotype and function of human antigen-presenting dendritic cells, Sci. Rep., 2017, 7, 14.

34 D. H. Craig, K. L. Schaubert, H. Shiratsuchi, J. Kan-Mitchell and M. D. Basson, Increased pressure stimulates aberrant dendritic cell maturation, Cell. Mol. Biol. Lett., 2008, 13, 260.

35 D. H. Craig, H. Shiratsuchi and M. D. Basson, Increased extracellular pressure provides a novel adjuvant stimulus 
for enhancement of conventional dendritic cell maturation strategies, Biochem. Biophys. Res. Commun., 2009, 387, 174.

36 M. I. Bonetti, L. Pieri, L. Domenici, S. Urbani, G. Romano, A. Aldinucci, C. Ballerini, M. Monici, R. Saccardi, V. Basile, A. Bosi and P. Romagnoli, Dendritic cells with lymphocytestimulating activity differentiate from human CD133 positive precursors, Blood, 2011, 117, 3983.

37 C. Wang, L. G. Xu, C. Liang, J. Xiang, R. Peng and Z. Liu, Immunological responses triggered by photothermal therapy with carbon nanotubes in combination with anti-CTLA-4 therapy to inhibit cancer metastasis, Adv. Mater., 2014, 26, 8154 .

38 S. A. Chechetka, Y. Yu, X. Zhen, M. Pramanik, K. Y. Pu and E. Miyako, Light-driven liquid metal nanotransformers for biomedical theranostics, Nat. Commun., 2017, 8, 19.

39 G. Lemperle, V. Morhenn and U. Charrier, Human histology and persistence of various injectable filler substances for soft tissue augmentation, Aesthetic Plast. Surg., 2003, 27, 354. 